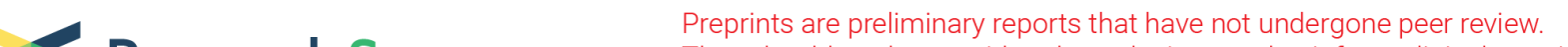 $\begin{array}{ll}\text { Research Square } & \begin{array}{l}\text { They should not be considered conclusive, used to inform clinical practice, } \\ \text { or referenced by the media as validated information. }\end{array}\end{array}$
}

\section{Periprosthetic Joint Infection - Comparison of Automated Multiplex-PCR Unyvero i60 ITI Cartridge System with Bacterial Culture and Real-Time PCR}

\section{Martin Luedemann ( $\sim$ m-luedemann.klh@uni-wuerzburg.de )}

Orthopaedische Klinik Koenig-Ludwig-Haus, University of Wuerzburg, Germany https://orcid.org/00000002-8401-1318

\section{Saskia Sulastyanto}

Orthopädische Klinik König-Ludwig-Haus: Orthopadische Klinik Konig-Ludwig-Haus

\section{Peter Raab}

Orthopädische Klinik König-Ludwig-Haus: Orthopadische Klinik Konig-Ludwig-Haus

\section{Christoph Schoen}

Institute for Hygiene and Microbiology, University of Wuerzburg

\section{Maximilian Rudert}

Orthopädische Klinik König-Ludwig-Haus: Orthopadische Klinik Konig-Ludwig-Haus

\section{Research article}

Keywords: Periprosthetic joint infection, multiplex-PCR, microbiological diagnosis, synovial fluid, $16 \mathrm{~S}$ rDNA PCR

Posted Date: May 4th, 2021

DOl: https://doi.org/10.21203/rs.3.rs-482098/v1

License: @ (i) This work is licensed under a Creative Commons Attribution 4.0 International License. Read Full License

Version of Record: A version of this preprint was published at Technology and Health Care on January 13th, 2022. See the published version at https://doi.org/10.3233/THC-213248. 


\section{Abstract}

Purpose: The evaluation of an automated-multiplex-PCR cartridge system for patients with suspicion of a periprosthetic joint infection (PJI) in comparison with conventional microbiological culture and 16S-rDNAPCR.

Methods: On suspicion of PJI synovial fluid specimen were taken preoperatively or periprosthetic tissue was collected intraoperatively. Microbiological analysis included conventional culture, 16S-rDNA-PCR and automated-multiplex-PCR (Unyvero-i60-ITI®). The European Bone and Joint Infection Society (EBJIS) criteria were used for PJI diagnosis. Positive and negative percent agreement was calculated. Total percentage agreement and Cohen`s kappa coefficient were calculated. Sensitivity, specificity and positive predictive value of conventional culture, 16S-rDNA-PCR and multiplex-PCR were calculated. 10 specimens of proved PJI were used as control group.

Results: 50 specimen were suitable for culture. 14 (28\%) were classified as PJI, 36 (72\%) were aseptic. Coagulase-negative staphylococci was the most frequent detected pathogen. Concordance-rate between $\mathrm{mPCR}$ and culture results was $75.6 \%$ with a Cohen Skof0.28. Conc or dance - ratebetweenmPCR and $16 S$ - rDNAwas82.9\%, Cohens kappa was 0.13. Concordance analysis between culture results and 16S-rDNA lead to a concordance-rate of $88.9 \%$. Cohen`s kappa was calculated with 0.6 . With regard to the microbiological culture as reference, sensitivity of the mPCR was 0.33 and specificity was 0.91 . Sensitivity and specificity of the 16S-rDNA-PCR was 0.55 and 0.97 . The positive predictive value was 0.57 for the $\mathrm{mPCR}$ and 0.83 for the $16 \mathrm{~S}-\mathrm{rDNA}-\mathrm{PCR}$.

Conclusion: Due to fair agreement between $\mathrm{MPCR}$ and conventional microbiological culture, the tested multiplex-PCR could be an additional instrument for the detection of PJI but is not superior over the conventional culture.

\section{Background}

Periprosthetic joint infection (PJI) is rare, but still a common and serious cause of failure in total hip- or knee arthroplasty $[1,2]$. The treating of this serious complication is an economic burden, linked with a prolonged inpatient stay, as well as higher morbidity and mortality $[3,4]$. Diagnosis of PJI is often difficult due to missing standardized definitions of and consent diagnostic algorithm in spite of different available tests. Clinical and radiological diagnosis remain subtle to differentiate between septic or aseptic loosening, biofilm-forming bacteria, and often culprit of PJI, adhere on abiotic artificial material and complicate diagnostic approaches $[5,6]$. A pre-operative identification of causing microorganisms and associated antimicrobial susceptibility is crucial for successful surgical and supportive local and systemic antibiotic treatment. Bacteria enclosed in an amorphic extracellular matrix, are frequently not detected by arthrocentesis and conventional microbiological culture, especially in low-grade PJI with low microbial burden or already given antibiotics [7, 8]. Over the past years, numerous attempts have been made to investigate and enhance diagnostic tools, such as sonication of removed implants or employing 
molecular techniques. Only a few studies have evaluated the diagnostic usefulness of polymerase chain reaction (PCR) in this context, especially concerning the function of multiplex-PCR assays comparing with conventional tissue culture. Orthopaedic departments, mostly with external microbiological laboratories are limited by infrastructural problems, such as biopsy shipment within 4 hours of collection. Another problem is the necessary length of time with a needed extended incubation of the biopsies in fluid enrichment broth for 10-14 days, otherwise slow growing microbes (e.g. Proprionibacterium spp. or small colony variants (SCV)) will not be detected $[9,10]$. Automated and simply operable multiplex-PCR systems are evolving for genotyping evaluation of bacteria. Advantages of these systems are a quick turnaround time, prompt detection within four to five hours of the typical PJI-relevant pathogens including genotyping resistance patterns and a higher sensitivity in cases of prior use of antibiotics. The Unyvero® i60 Implant and Tissue Infection Cartridge is a commercially available quick molecular assay, which can identify a narrow list of pathogens among the most frequently implicating PJI. Here we inform of our clinical experience comparing the automated multiplex-PCR Unyvero i60 ITI (Implant and Tissue Infection) cartridge (U-ITI) application (Curetis AG, Holzgerlingen, Germany) with conventional culture and with a $16 \mathrm{~S}$ rDNA PCR assay in patients with suspected PJI.

\section{Methods}

\section{Study design}

This retrospective cohort study was done in a university medical hospital, specialized in advanced orthopedic surgery. The study protocol was reviewed and approved by the institutional ethics committee of the Medical University of Wuerzburg (20200316 01) and was conducted in accordance with the declaration of Helsinki. The PCR-results were not relayed to the treating physicians and so had no impact on any therapeutic decision.

\section{Study population}

Between June 2015 and April 2016 fifty-one consecutive patients were included, minimum age 18 years, with suspected periprosthetic joint infection. All joint aspirations had been performed in connection with routine clinical pre-operative diagnosis by an orthopedic surgeon in conformity with the described standardized strictly aseptic technique in our institution [11]. Any antibiotic therapy had to be stopped at least 2 weeks before arthrocentesis. All periprosthetic tissue samples had been taken throughout the surgical procedure for routine microbiological culture. Identifiable contamination of the joint fluid, impurity during transport to the microbiological laboratory as well as invalid test results of the multiplex PCR, or incomplete data set were defined as exclusion criteria. All clinical information was acquired from the medical records. A periprosthetic joint infection was defined in accordance to the criteria, proposed by the European Bone and Joint Infection Society (EBJIS) [12]. We used further 10 frozen specimen of known bacterial infections from the Institute for Hygiene and Microbiology, University of Wuerzburg as a retrospective control. 
Aspirated synovial fluid $(2,5 \mathrm{ml})$ was transferred in a sterile vial to the Institute for Hygiene and Microbiology (University of Würzburg, Germany) within $2 \mathrm{~h}$ at room temperature in accordance with current guidelines $[13,14]$. Cultivation and identification of bacteria were performed according to standard operation procedures (SOPs) based on recommendations by the German Society for Hygiene and Microbiology (DGHM) $[15,16]$. Shortly, for aerobic and anaerobic culture all samples were incubated in brain heart infusion (BHI) broth, thioglycolate bouillon (Becton Dickinson, New Jersey, USA), and on Columbia blood agar (BioMérieux, Marcy L`Etoile, France), chocolate agar (Becton Dickinson), and Schaedler-agar (with vitamin K1) (Becton Dickinson) plates. BHI broth, Columbia blood agar and chocolate agar plates were incubated at $36^{\circ} \mathrm{C}$ with $5 \% \mathrm{CO}_{2}$ for 48 hours and inspected visually after 24 and 48 hours. Schaedler-agar plates were incubated under anaerobic conditions for 4 days at $36^{\circ} \mathrm{C}$, and the thioglycolate bouillon was incubated at $36^{\circ} \mathrm{C}$ with $5 \% \mathrm{CO}_{2}$ and inspected visually every day for 14 days. In the case of turbidity, thioglycolate bouillons were immediately subcultured on Schaedler agar (followed by anaerobic incubation) and chocolate agar (followed by aerobic incubation) plates. In case of no visible growth thioglycolate bouillons were routinely subcultured on days 7 and 14 on Schaedler agar (followed by anaerobic incubation) and chocolate agar (followed by aerobic incubation) plates to exclude the growth of any viable bacteria in the sample. Mass spectrometry (Vitek® MS, BioMérieux, Marcy

L'Etoile, France) was used for pathogen identification of individual clones grown on agar plates according to the manufacturer's recommendations and susceptibility testing was routinely performed using Vitek ${ }^{\circledR} 2$ (BioMérieux) and/or alternatively with the European Committee on Antimicrobial Susceptibility Testing (EUCAST) disc diffusion method and explained according to the EUCAST clinical breakpoints - bacteria (v 5.0) (https://www.eucast.org).

\section{Bacterial species detection via 16S-rDNA PCR}

Bacterial species detection and identification from aspirated synovial fluid samples via 16S rDNA PCR and sequencing was performed as described previously [17]. All PCR assays were performed with strict precautions in a highly standardized manner to avoid any DNA cross-contamination in line with current DGHM guidelines $[18,19]$. Sequencing of positive samples was performed at LGC Biotech. Only sequences $>700$ bases were used for nucleotide sequence similarity searches against RDPII [20, 21] (https://rdp.cme.msu.edu/), BIBI [22] (http://pbil.univ-lyon1.fr/bibi/), Greengenes [23] (http://greengenes.lbl.gov) and GenBank [24] (www.ncbi.nlm.nih.gov/genbank/) databases. Species identification was performed on the basis of sequence similarity according the recommendations given by She et al. [25]. The analytical sensitivity of the method was adjusted to $>10^{2} \mathrm{rDNA}$ copies $/ \mathrm{mL}$ to reduce false-positive results.

\section{Multiplex PCR cartridge system}

The Unyvero i60 device (Curetis, Holzgerlingen, Germany) with the employed Implant and Tissue Infection cartridge application (U-ITI) allows a semi quantitative DNA determination based on the simultaneously performance of eight multiplex PCR (MPCR) reactions and detection of up to 114 pathogen associated nucleic acids and resistance markers of specific pathogens causing PJI in fluid samples (Table 1). All synovia fluid specimen were processed with the Unyvero i60 ITI application according to the 
manufacture`s protocol as described earlier [26, 27]. The aspirated synovial fluid was centrifuged and $180 \mu \mathrm{l}$ of the pellet was transferred into the Unyvero sample tube, containing specific lysis reagents. The sample lysis consists of thermal, mechanical, chemical and enzymatic treatment for 30 minutes. After lysing, the specimen was processed in the i60 ITI cartridge, pre-filled with reagents for DNA purification, PCR primers and probes for array hybridization. A result was classified as positive if at least one of the analytes (pathogens) reached the threshold of positivity $\left(10^{4} \mathrm{DNA}\right.$ fragments/pathogen/ml). A synthetic gene without significant homology to known sequences was co-processed in every of the eight PCRchambers as an internal control to verify DNA purification, PCR and array hybridization.

\section{Statistical analysis}

Positive and negative percent agreement between conventional culture, 16S-rDNA PCR and multiplex PCR was calculated. To measure overall agreement, total percentage agreement and Cohen`s kappa coefficient were determined. Sensitivity, specificity and positive predictive value (PPV) of conventional culture, 16S-rDNA PCR and multiplex PCR were calculated. A p-value $<0,05$ was considered significant. Statistical analyses were performed using the program package $\mathrm{R}$ (Version 3.5.0., http://www.rstatistics.com). Cohen`s kappa was categorized as follows: $<0$ : no agreement, 0,01-0,2:none to slight, $0,21-0,4$ : fair, 0,41-0,6: moderate, $0,61-0,8$ : substantial and 0,81-1,0: almost perfect.

\section{Results}

\section{Patient demographics and infection classification}

Specimen and clinical details were acquired from 55 patients of whom four had to be excluded because of inappropriate specimen processing. A total of 51 patients were included with suspected periprosthetic infection. The median age of the 27 female and 24 male patients was 67 (range, 43-87) years, involving $12(23,5 \%)$ hip, 37 (72,5\%) knee and $2(4 \%)$ shoulder prostheses. Thirty samples were taken preoperatively, 21 specimen were gained intra-operatively. 38 samples were fluid punctures, 13 were tissue. (Table 2). All of the additional 10 control-specimen were primary taken intraoperatively with microbiological proven infection.

Of all 51 patients, 1 specimen was not suitable for culture. Consistent with the EBJIS-criteria, fourteen patients (28\%) were classified with PJI and 36 (72\%) with aseptic failure. 16S rDNA PCR and multiplexPCR Unyvero were carried out for all patients, but 5 samples of each method couldn't be analyzed successfully.

\section{Study group}

\section{Microbiological characteristics}

Of the infected patients, 13 (92.9\%) were monomicrobial in culture, the remaining 1 (7.1\%) were positive for two (polymicrobial infections) in culture. Among the total of 15 isolated microorganisms in the studygroup, the detected pathogens were: coagulase-negative staphylococci $(n=5)$, Streptococcus agalactiae 
$(\mathrm{n}=2)$, Proteus vulgaris $(\mathrm{n}=2)$, Parvimonas micra $(\mathrm{n}=2)$, Pseudomonas aeruginosa $(\mathrm{n}=1)$, Staphylococcus lugdunensis $(\mathrm{n}=1)$, Micrococcus luteus $(\mathrm{n}=1)$ and Enterococcus faecalis $(\mathrm{n}=1)$.

\section{Concordance analysis between MPCR (Unyvero®) and culture results}

Overall, 34 out of the 45 valid samples were concordant with respect to pathogen identification, showing a concordance-rate of $75.6 \%$. 4 out of 12 positive microbial cultures were also detected by the mPCR, while 30 specimen remained sterile in culture and mPCR. The Unyvero ${ }^{\circledR} \mathrm{mPCR}$ showed 8 false negative and 3 false positive results (Table 3). Cohen`s kappa was calculated with 0.28.

\section{Concordance analysis between MPCR (Unyvero®) and 16S- rDNA}

41 samples were comparable for mPCR and 16S-rDNA, in 34 specimen there was an agreement between the results. The concordance-rate was $82.9 \%$ (Table 4). Cohen`s kappa was 0.13 .

\section{Concordance analysis between culture results and 16S- rDNA}

40 out of 45 interpretable specimen showed concordance for identification of the causative pathogen, leading to a concordance-rate of $88.9 \% .5$ out of 9 comparable positive cultures were also detected by the 16S-rDNA PCR, while 35 samples remained sterile in 16S-rDNA PCR and microbiological culture. The 16SrDNA PCR showed 4 false negative and 1 false positive result (Table 5). Cohen`s kappa was calculated with 0.6 .

With regard to the microbiological culture as reference, the sensitivity of the Unyvero $\AA$ mPCR was 0.33 $(3 / 12)$ and specificity was $91 \%$. A sensitivity and specificity of the 16S-rDNA PCR were calculated with $0.55(5 / 9)$ and $0.97(30 / 33)$. The positive predictive value was calculated with 0.57 for the Unyveroß mPCR and with 0.83 for the 16S-rDNA-PCR.

\section{Control group:}

The control group implied 10 samples from proven tissue infections with Staphylococcus aureus (2x), Staphylococcus epidermidis (1x), Escherichia coli (3x), Proprionibacterium acnes (2x) and Enterococcus faecalis (2x). While the Unyvero ${ }^{\circledR} \mathrm{mPCR}$ was carried out for all control specimen, the 16S-rDNA PCR was feasible only in 6 cases, due to too few material.

Concordance between mPCR (Unyvero®) and culture results: In the control-group $(n=10) 2$ out of ten samples were concordant for the identification of the pathogen, the mPCR detected Proprionibacterium acnes in one case instead of $E$. coli in the culture. In one case, the MPCR detected $P$. acnes, while $E$. coli was detected by the conventional culture. The other 6 specimen ( $2 \times$ E. coli, 3x P. acnes, $1 \times$ S. epidermidis) remained without pathogen identification with the $\mathrm{MPCR}$. 
Concordance between mPCR (Unyvero®) and 16S-rDNA: In the control-group 4 out of 6 comparable samples showed concordant results (66.7\%). The mPCR detected $P$. acnes in one case, whereas 16SrDNA PCR detected Methylobacterium spp..

Concordance between culture results and 16S-rDNA: In the control-group were 6 comparable results. 1 positive culture (S. aureus) was also detected by the 16S-rDNA-PCR (16.7\%), which showed a Methylobacterium spp. and Anaerococcus spp. as false positive results in 2 other measurements. The 3 other specimen ( $2 \times$ E. coli and $1 \times$ S. aureus) remained without detection through the 16S-rDNA-PCR.

In the control-group, the sensitivity of the Unyvero® mPCR was $30 \%(3 / 10)$ and specificity was $91 \%$. Sensitivity and specificity of the 16S-rDNA PCR were calculated with 55\% (5/9) and 97\% (30/33). The positive predictive value was $1 \%$ for both the Unyvero ${ }^{\circledR}$ mPCR and the $16 \mathrm{~S}-\mathrm{rDNA}-\mathrm{PCR}$.

\section{Discussion}

Clinical, radiological and microbiological criteria have to be taken into account for the evidence of periprosthetic joint infection. This multidisciplinary approach is necessary, as there is still no reference diagnostic standard [28]. While Erythrocyte Sedimentation Rate (ESR) or serum C-reactive Protein (CRP) have not proved as secure preoperative infection markers, microbiological culture of synovial fluid or tissue samples is the present diagnostic gold standard, although molecular methods, biomarkers, such as alpha-defensin or sonication of the removed prosthesis are suggested to improve diagnostic performance $[29,30]$. Varying specimen, such as synovial fluid, biopsies from bone or periprosthetic tissues or the sonication fluid from the removed prosthesis itself show different performances in different diagnostic procedures and complicate the comparison between existing studies [31]. Culture has a limited sensitivity and specificity due to previous antibiotic usage or contaminating skin-microorganisms, which are difficult to distinguish from true pathogens. Before conventional culture results are available, often a long time period of up to 14 days is needed for a conclusive result. Therefore, PCR-techniques have been focused on in recent years, having shown to detect PJI and providing information about causative pathogen in a timely manner [32]. Here, we inform of a investigation comparing conventional microbiological culture with two different PCR-methods using prospectively collected specimen from patients with suspected periprosthetic joint infection. In the present study the overall concordance rate between the MPCR and conventional culture with respect to the detection of any bacterial species was $75.6 \%$. These results seem to be consistent with previous studies on PCR-techniques. Morgenstern et al. reports a concordance rate of $82 \%$ in 116 patients of septic and aseptic cases [33]. This is also in accordance with the results reported by Sigmund et al., showing a concordance rate between culture and mPCR about $85.6 \%$ in 90 patients [32]. Borde et al. conducted a investigation with 54 patients by comparing culture with 16S-rDNAPCR and $\mathrm{MPCR}$, reporting a concordance rate of $82 \%$ concerning culture and $\mathrm{mPCR}$ [34]. mPCR results in our study were also compared to the 16S-rDNA-PCR, and notwithstanding differences in the identity of the bacterial species detected an overall concordance rate of $82.9 \%$ was determined. Borde et al. report a concordance rate between culture and 16S-rDNA-PCR about $96 \%$ in their study. Our findings seem to be accordant with the existing data on PCR-techniques regarding diagnosis of PJI. Hirschebeth et al. report a 
sensitivity of $66.7 \%$ and a specificity of $100 \%$ for the Unyvero mPCR when compared to cultural method in a study with 62 specimen from 31 patients [35]. In our study we calculated a sensitivity of the Unyvero $\AA \mathrm{mPCR}$ of $33 \%$ and specificity of $91 \%$. The sensitivity and specificity of the $16 \mathrm{~S}$-rDNArDNA PCR was calculated with $55 \%$ and $97 \%$. Using conventional culture as reference, the positive predictive value for the presence of bacterial DNA in the sample and thus infection was calculated with $57 \%$ for the Unyvero ${ }^{\circledR} \mathrm{MPCR}$ in our study, while Hirschebeth et al. reports $100 \%$ using sonication and synovial fluids [35]. Likewise, 16S-rDNA-PCR can identify pathogens in synovial fluid with a sensitivity and specificity of $84 \%$ and $89 \%$, in sonication fluid of $81 \%$ and $96 \%$ as well as a high sensitivity and specificity in patients on antibiotics [36]. Restrictions of this sensitive technique are current its higher cost compared to culture and the susceptibility to contamination leading to false positive findings. In our study, the 16S-rDNA-PCR detected a Methylobacterium in one case, while the Unyvero MPCR remained without bacterial detection and the culture showed S. epidermidis. As well as its normal habitats in soil and water, Methylobacterium has also been recognized as a contaminant of DNA extraction kit reagents, which may result in its erroneous appearance in nucleic acid amplification techniques [37]. To reduce the false-positive rates, attempts have been made by establishing a detection threshold, but this could decrease the sensitivity of these tests [34,38]. In addition, also a low bacterial inoculum found in positive conventional culture samples might be probably linked to a negative PCR-result [39]. Another potential limitation of the commercially $\mathrm{mPCR}$ test is to narrow only on the identification of a pre-selected list of causative pathogens. In particular, the Unyvero $160 \mathrm{ITI} \circledast$ is a mPCR dedicated to the diagnosis of prosthetic joint infections, considering only pathogens that are considered to the most relevant in causing PJI, including P. acnes. In our study only $13.3 \%(2 / 15)$ were positive in culture for bacteria not included in the Unyvero i60 ITI-panel (1x Micrococcus luteus, 1x Parvimonas micra). The simultaneous use of mPCR and culture could be a more sensitive diagnostic tool. At present, the mPCR cannot yet replace conventional microbiological culture, which has still to be considered as the standard of care in preoperative diagnostic of periprosthetic infection.

Our study has therefore limitations. To start with the overall number of patients $(n=51)$ and the number of culture-positive cases $(n=14)$ is rather low. Hence the concordance rate of $75.6 \%$ is predominantly based on concordant negative results $(n=30)$. Future investigations, with higher count of patients will be needed to more reliably establish sensitivity and specificity of mPCR or 16S-rDNA-PCR. Second, our study group contains different locations of possible infected implants including hip, knee and shoulder, as well as different types of specimen, such as synovial fluid or periprosthetic tissue. Forthcoming studies should focus on special locations and identically specimen.

\section{Conclusion}

In conclusion, with regard to diagnosis of prosthetic joint infection, our results gave no evidence of superiority of the tested mPCR compared to conventional culture. The multiplex-PCR-System provides the advantage of a short turnaround time (within 5 hours), whereas conventional culture requires several days for growth. Conventional microbiological culture with antibiotic susceptibility testing should be performed in first-line, especially to detect pathogens, which cannot be detected by the tested Unyvero i60 
ITI ${ }^{\circledR}$ cartridge. Molecular methods might be useful as second line for complex cases with difficult to detect microorganisms and complement conventional culture. The used mPCR-technique did not provide extra information than that acquired from culture. Further improvement of the mPCR, for example with inclusion of additional primers is desirable, prospective trials are necessary.

\section{Abbreviations}

$\mathrm{BHI}$

brain heart infusion

CRP

C-reactive Protein

DGHM

German Society for Hygiene and Microbiology

EBJIS

European Bone and Joint Infection Society

EUCAST

European Committee on Antimicrobial Susceptibility Testing

ITI

Implant and Tissue Infection

$\mathrm{m}-\mathrm{PCR}$

multiplex-polymerase chain reaction

PJI

periprosthetic joint infection

PCR

polymerase chain reaction

SCV

small colony variants

SOP

standard operation procedure

\section{Declarations}

\section{Ethical Approval and consent to participate:}

The study protocol was 'retrospectively registered', reviewed and approved by the institutional ethics committee of the Medical University of Wuerzburg (20200316 01) and was done in accordance with the declaration of Helsinki (see attached uploaded file No. 4 - Compliance with Ethical Standards). All authors declare no potential conflicts of interests. Each author declares that (s)he has no commercial associations (e.g. consultancies, stock ownership, equity interest, patent/licensing arrangements, etc.) that might pose a conflict of interest in connection to the submitted article. Consent to Participate: All participants gave written consent to participate. 
Consent for publication:

Not applicable

Availability of data and materials:

The datasets used and analyzed during the current study are available from the corresponding author on reasonable request.

Competing interests:

All authors certify that they have no affiliations with or involvement in any organization or entity with any financial interest or non-financial interest in the subject matter or materials discussed in this manuscript.

Funding:

This publication was supported by the Open Access Publication Fund of the University of Wuerzburg. The manufacturer (Curetis AG) provided Unyvero reagents and instruments.

Authors Contributions:

All authors contributed to the study conception and design. Material preparation, data collection and analysis were performed by Saskia Sulastyanto and Christoph Schoen. Martin Luedemann wrote the first draft of the manuscript and all authors commented on previous versions of the manuscript. All authors read and approved the final manuscript.

\section{References}

1. Bozic KJ. The epidemiology of revision total knee arthroplasty in the United States. Clin Orthop Relat Res. 2010;468:45-51.

2. Bozic KJ. The epidemiology of revision total hip arthroplasty in the United States. J Bone Joint Surg Am. 2009;91:128-33.

3. Kurtz SM. Future clinical and economic impact of revision total hip and knee arthroplasty. J Bone Joint Surg Am. 2007;89:144-51.

4. Bozic KJ. The impact of infection after total hip arthroplasty on hospital and surgeon resource utilization. J Bone Joint Surg Am. 2005;87:1746-51.

5. Portillo ME. Sonication versus vortexing of implants for diagnosis of prosthetic joint infection. J Clin Microbiol. 2013;51:591-4.

6. Tzeng A. Treating periprosthetic joint infections as biofilms: key diagnosis and management strategies. Diagn Microbiol Infect Dis. 2015;81:192-200.

7. Berbari EF. Culture-negative prosthetic joint infection. Infect Dis. 2007;45:1113-9. 
8. Trampuz A. New methods for the diagnosis of implant-associated infections. Rev Med Suisse. 2010;6:731-4.

9. Parvizi J. Proceedings of the International Consensus on Periprosthetic Joint Infection. Bone Joint J. 2013;95-B:1450-1452.

10. Schäfer P. Prolonged bacterial culture to identify late periprosthetic joint infection: a promising strategy. Clin Infect Dis. 2008;47:1403-9.

11. Lüdemann CM. Diagnosis of periprosthetic hip infections. Oper Orthop Traumatol. 2015;27:237-51.

12. McNally M. The EBJIS definition of periprosthetic joint infection. Bone Joint J. 2021;103-B:18-25.

13. Baron EJ. A guide to utilization of the microbiology laboratory for diagnosis of infectious diseases: 2013 recommendations by the Infectious Diseases Society of America (IDSA) and the American Society for Microbiology (ASM). Clin Infect Dis. 2013;57:485-8.

14. Miller JM. A Guide to Utilization of the Microbiology Laboratory for Diagnosis of Infectious Diseases: 2018 Update by the Infectious Diseases Society of America and the American Society for Microbiology. Clin Infect Dis. 2018;67:813-6.

15. Podbielski A. Mikrobiologisch-infektiologische Qualitätsstandards. 18th ed. Munich: Elsevier; 2014.

16. Podbielski A. Mikrobiologisch-infektiologische Qualitätsstandards. 19th ed. Munich: Elsevier; 2014.

17. Liese JG. Changes in the incidence and bacterial aetiology of paediatric parapneumonic pleural effusions/empyema in Germany, 2010-2017: a nationwide surveillance study. Clin Microbiol Infect. 2019;25:857-64.

18. Apfalter P. In-house nucleic acid amplification assays in research: how much quality control is needed before one can rely upon the results? J Clin Microbiol. 2005;43:5835-41.

19. Reischl U. Mikrobiologisch-infektiologische Qualitätsstandards. 1st ed. Munich: Elsevier; 2011.

20. Maidak BL. The RDP-II (Ribosomal Database Project). Nucleic Acids Res. 2001;29:173-4.

21. Cole JR. "The ribosomal database project (RDP-II): introducing myRDP space and quality controlled public data." Nucleic Acids Res Database issue (2007): D169-72. doi:10.1093/nar/gkl889.

22. Devulder G. BIBI, a bioinformatics bacterial identification tool. J Clin Microbiol. 2003;41:1785-7.

23. DeSantis TZ. Greengenes, a chimera-checked 16S rDNA gene database and workbench compatible with ARB. Appl Environ Microbiol. 2006;72:5069-72.

24. Sayers EW. GenBank. Nucleic Acids Res. 2020;48,D84-D86, doi:10.1093/nar/gkz956.

25. She RC, Simmon KE, Petti CA. Diagnostic principles and practice. In: Persing DH, editor. Molecular Microbiology. Washington, DC: ASM Press; 2011. pp. 479-89.

26. Curetis@. 2017, Unyvero ITI Application Manual, Item. No. 00207, Rev. 4.0 - EN-G2, 2017.

27. Renz N. Performance of automated multiplex PCR using sonication fluid for diagnosis of periprosthetic joint infection: a prospective cohort. Infection. 2017;45:877-84.

28. Osmon DR. Diagnosis and Management of Prosthetic Joint Infection: Clinical Practice Guidelines by the Infectious Diseases Society of America. Clin Infect Dis. 2013;56:e1-25. Doi:10.1093/cid/cis803. 
29. Corvec S. Epidemiology and new developments in the diagnosis of prosthetic joint infection. Int $J$ Artif Organs. 2012;35:923-34.

30. Deirmengian C. Diagnosing Periprosthetic Joint Infection Has the Era of the Biomarker Arrived? Clin Orthop Relat Res. 2014;472:3254-62.

31. Lévy PY. The role of molecular diagnostics in implant-associated bone and joint infection. Clin Microbiol Infect. 2012;18:1168-75.

32. Sigmund IK. Multiplex PCR Unyvero i60 ITI application improves detection of low-virulent microorganisms in periprosthetic joint infections. Int Orthop. 2019;43:1891-8.

33. Morgenstern C. Synovial Fluid Multiplex PCR Is Superior to Culture for Detection of Low-Virulent Pathogens Causing Periprosthetic Joint Infection. Diagn Microbiol Infect Dis. 2018;90:115-9.

34. Borde JP. Diagnosis of Prosthetic Joint Infections Using UMD-Universal Kit and the Automated multiplex-PCR Unyvero i60 ITI(®) Cartridge System: A Pilot Study. Infection. 2015;43:551-60.

35. Hirschebeth GTR. Unyvero i60 Implant and Tissue Infection (ITI) Multiplex PCR System in Diagnosing Periprosthetic Joint Infection. J Microbiol Methods. 2016;121:27-32.

36. Izakovicova P. Periprosthetic joint infection: current concepts and outlook. EFORT Open Rev. 2019;4:482-94.

37. Salter SJ. Reagent and laboratory contamination can critically impact sequence-based microbiome analyses. BMC Biol. 2014;12:87. doi:10.1186/s12915-014-0087-z.

38. Tunney MM. Detection of prosthetic hip infection at revision arthroplasty by immunofluorescence microscopy and PCR amplification of the bacterial 16S rDNA gene. J Clin Microbiol. 1999;37:328190.

39. Prieto-Borja L. Evaluation of a commercial multiplex PCR (Unyvero $i 60 \AA$ ) designed for the diagnosis of bone and joint infections using prosthetic-joint sonication. Enferm Infecc Microbiol Clin. 2017;35:236-42.

\section{Tables}

Due to technical limitations, table 1 to 5 is only available as a download in the Supplemental Files section.

\section{Supplementary Files}

This is a list of supplementary files associated with this preprint. Click to download.

- Tables20210222.docx 